\title{
STRUCTURAL COVARIANCE OF THE NEOSTRIATUM WITH REGIONAL GRAY MATTER VOLUMES
}

Soriano-Mas, C. ${ }^{1,2^{\star}}$; Harrison, B.J. ${ }^{3}$; Pujol, J. ${ }^{4}$; López-Solà M. ${ }^{4}$; Hernández-Ribas, R. ${ }^{1,2}$; Alonso, P. ${ }^{1,2,5}$; Contreras-Rodríguez, O. ${ }^{4}$; Giménez, M. ${ }^{4}$; Blanco-Hinojo, L. ${ }^{4,6}$; Ortiz, H. ${ }^{4}$; Deus, J. ${ }^{4,7}$; Menchón, J.M. ${ }^{1,2,5} ;$ and Cardoner, N. ${ }^{1,2,5}$

1. Department of Psychiatry, Bellvitge University Hospital-IDIBELL, Barcelona, Spain; 2. Carlos III Health Institute, Centro de Investigación Biomédica en Red de Salud Mental (CIBERSAM), Spain; 3. Melbourne Neuropsychiatry Centre, Department of Psychiatry, The University of Melbourne \& Melbourne Health, Australia; 4. CRC Hospital del Mar, Barcelona, Spain; 5. Department of Clinical Sciences, School of Medicine, University of Barcelona, Barcelona, Spain; 6. Fundació IMIM-PRBB, Barcelona, Spain; 7. Department of Clinical and Health Psychology, Autonomous University of Barcelona, Barcelona, Spain.

${ }^{*}$ Corresponding author:

Dr. Carles Soriano-Mas, PhD

Psychiatry Department, Bellvitge University Hospital-IDIBELL

Feixa Llarga s/n, 08907, Barcelona, Spain

E-mail: csoriano@idibell.cat

Telephone: +34 932607500 (ext. 2864)

Fax: +34932607658

Keywords: Structural covariance, brain anatomy, structural plasticity, brain connectivity, neostriatum, neuroimaging, magnetic resonance imaging 


\section{Abstract}

The caudate and putamen nuclei have been traditionally divided into dorsal and ventral territories based on their segregated patterns of functional and anatomical connectivity with distributed cortical regions. Activity-dependent structural plasticity may potentially lead to the development of regional volume correlations, or structural covariance, between the different components of each cortico-striatal circuit. Here, we studied the whole-brain structural covariance patterns of four neostriatal regions belonging to distinct cortico-striatal circuits. We also assessed the potential modulating influence of laterality, age and gender. T1-weighted three-dimensional magnetic resonance images were obtained from ninety healthy participants (50 females). Following data pre-processing, the mean signal value per hemisphere was calculated for the 'seed' regions of interest, located in the dorsal and ventral caudate and the dorsal-caudal and ventral-rostral putamen. Statistical Parametric Mapping was used to estimate whole-brain voxel-wise structural covariance patterns for each striatal region, controlling for the shared anatomical variance between regions in order to obtain maximally specific structural covariance patterns. As predicted, segregated covariance patterns were observed. Age was found to be a relevant modulator of the covariance patterns of the right caudate regions, while laterality effects were observed for the dorsal-caudal putamen. Gender effects were only observed via an interaction with age. The different patterns of structural covariance are discussed in detail, as well as their similarities with the functional and anatomical connectivity patterns reported for the same striatal regions in other studies. Finally, the potential mechanisms underpinning the phenomenon of volume correlations between distant corticostriatal structures are also discussed. 


\section{Introduction}

Although overall brain volume acquires up to seventy-five percent of its adult weight by the age of two years (Giedd et al. 1996), changes in regional brain structure continue throughout life (Yakolev and Lecours 1967; Huttenlocher 1979; Pujol et al. 1993). Moreover, regional volumes are only partially dependent on overall brain volume (Allen et al., 2002), and although the latter undergoes strong genetic control, there are a number of non-genetic factors that modulate regional brain volumes (Kennedy et al. 1998; Caviness et al. 1999). Non-invasive neuroimaging techniques, such as structural magnetic resonance imaging (MRI), have enabled the identification of some of these factors, such as activity-dependent structural plasticity (Butz et al. 2009), which reflects changes in regional anatomy as a function of the recent history of activity within a given region (Draganski et al. 2004; May and Gaser 2006). In this sense, the existence of functional brain networks of synchronously activated regions may be expected to result in correlated gray matter volumes across distant structures (Seeley et al. 2009).

In general, studies assessing volume correlations, or structural covariance, between distant brain regions have shown that homotopic and functionally related regions are significantly correlated (Andrews et al. 1997; Mechelli et al. 2005; Zielinski et al. 2010), and that such correlations may be differentially altered in brain disorders (Portas et al. 1998; Wright et al. 1999; Pujol et al. 2004; Mitelman et al. 2005, a, b, c; Cardoner et al. 2007; Modinos et al. 2009; Seeley et al. 2009; Xu et al. 2009; Kasparek et al. 2010). Although several methods are appropriate for structural covariance assessment (see, for instance, Xu et al. 2009), currently the 'seed-based' region-of-interest approach is the most widely used. This method is typically used to characterize voxel-wise structural covariance patterns across the whole brain with a selected 'seed' region that is considered to represent an important anatomical component of a broader functional network. The idea of estimating structural covariance in such a manner is roughly equivalent to functional MRI and diffusion tensor imaging (DTI) studies of functional and anatomical (i.e., white matter) connectivity, respectively. 
Since their initial description (Alexander et al. 1986), the so-called cortico-striatal circuits have been extensively studied both in basic and clinical neuroscience given the multiplicity of functions they support and their relevance for different disorders. The caudate nucleus and putamen (neostriatum) have been traditionally divided into dorsal and ventral functional territories based on their known anatomical connectivity with distributed cortical regions, and, in particular, the frontal cortex (Alexander et al. 1986; Lawrence et al. 1998; Nakano et al. 2000). The territories have come to be recognized as distinct components of multiple distributed 'cortico-striatal circuits' or networks implicated in different cognitive, motor ('dorsal') and emotional-motivational ('ventral') aspects of behavior (Haber 2003). Importantly, fMRI and DTI studies in humans have validated this dorsal-ventral distinction by mapping the functional and anatomical connectivity of caudate and putamen subregions (Lehéricy et al. 2004; Postuma and Dagher 2006; DiMartino et al. 2008; Draganski et al. 2008).

Structural covariance of the neostriatum has also been explored, with results supporting the existence of dorsal and ventral cortico-striatal networks and, more broadly, the idea that functionally related regions are indeed correlated in volume (Cohen et al. 2008; Colibazzi et al. 2008). However, due to differences in the precise neostriatal regions assessed in these studies, it has until now been difficult to compare their results directly. Differences in the location and size of selected regions are expected to have a relevant influence on resulting covariance or connectivity maps, in view of the known compression and partial overlap of such networks within small basal ganglia structures (Haber 2003). Additionally, as strong volumetric correlations are typically observed between closely located brain regions, the structural covariance patterns obtained from different striatal territories have proved to be somewhat unspecific. Such a problem might well be overcome by a firm statistical control of the anatomical variance shared between the different striatal territories under study.

The aim of the present study was to assess whole-brain structural covariance patterns of four distinct neostriatal territories: the dorsal and ventral caudate nucleus, and the dorsal-caudal and ventral-rostral putamen. We chose our anatomical coordinates on the basis of several recent functional connectivity studies that have mapped the putative corticostriatal networks in healthy 
participants (DiMartino et al. 2008; Kelly et al. 2009) and patients with neuropsychiatric disorders (Harrison et al. 2009; Kwak et al. 2010; DiMartino et al. 2011; Fitzgerald et al. 2011; Sakai et al. 2011). We controlled for the shared variance between our seed regions to obtain maximally specific structural covariance patterns for each of the regions studied. For each region, the potential modulating influence of age and gender, and the effects of laterality, were also investigated.

\section{Materials and methods}

\section{Subjects}

Ninety healthy volunteers participated in the study (50 women, mean \pm SD age $=31.9 \pm 10.85$ years, range $20-58$ years). Subjects were selected as healthy controls to participate in ongoing projects concerning structural brain alterations in psychiatric disorders. A detailed medical history was recorded and a structured clinical interview was administered in order to exclude subjects with current or past psychiatric, neurological or other relevant medical disorders, or contraindications to MRI. Post-scanning, subjects' data were also excluded if brain images proved to be abnormal upon visual inspection, although no subjects were actually excluded for this reason. All participants gave written informed consent to participate in the study, which was performed in accordance with the ethical standards laid down in the 1964 Declaration of Helsinki and approved by the institutional review board of the Bellvitge University Hospital, Barcelona.

\section{Data acquisition and pre-processing}

Images were acquired with a 1.5-T Signa Excite system (General Electric, Milwaukee, Wisconsin) equipped with an 8-channel phased-array head coil. A high-resolution T1-weighted anatomical image was obtained for each subject using a 3-dimensional fast spoiled gradient inversion-recovery prepared sequence with 130 contiguous slices (TR, 11.8 milliseconds; TE, 
4.2 milliseconds; flip angle, $15^{\circ}$; field of view, $30 \mathrm{~cm}$; 256x256 pixel matrix; slice thickness, 1.2 $\mathrm{mm})$.

Imaging data were transferred and processed on a Microsoft Windows platform using a technical computing software program (MATLAB 7; The MathWorks Inc, Natick, Mass) and Statistical Parametric Mapping software (SPM5; The Wellcome Department of Imaging Neuroscience, London, UK). Following the inspection of image artifacts, image preprocessing was performed with the VBM5 toolbox (http://dbm.neuro.uni-jena.de/vbm/). Briefly, native-space MRIs were segmented and normalized to the SPM-T1 template by means of the unified segmentation approach (Ashburner and Friston 2005). A hidden Markov random field model was applied to minimize the noise level of resulting gray matter segments, which were resliced to a final voxel size of $1 \mathrm{~mm}^{3}$. Additionally, the Jacobian determinants derived from the spatial normalization were used to modulate image voxel values to restore volumetric information (Good et al. 2001). Finally, images were smoothed with a $12 \mathrm{~mm}$ full-width at half maximum (FWHM) isotropic Gaussian kernel.

\section{Seed volumes extraction}

To assess the structural covariance pattern of each specific striatal subdivision, we extracted individual gray matter volumes from eight seed-regions of interest (four per hemisphere). Our approach aimed to segregate the structural covariance patterns of dorsal and ventral regions of the caudate and putamen nuclei. This approach was based on recent functional connectivity studies (DiMartino et al. 2008; Harrison et al. 2009) using the dorso-ventral boundaries initially proposed by Postuma and Dagher (2006). For each region, seeds were defined with the MarsBar region-of-interest toolbox (Brett et al. 2002) as $3.5 \mathrm{~mm}$ radial spheres centered at the following bilateral Montreal Neurological Institute (MNI) coordinates (see Fig. 1): (i) dorsal caudate $[x( \pm)=13, y=15, z=9]$; (ii) ventral caudate, involving the nucleus accumbens $[x( \pm)=9$, $y=9, z=-8]$; (iii) dorsal caudal putamen $[x( \pm)=28, y=1, z=3]$; and (iv) ventral rostral putamen $[x( \pm)=20, y=12, z=-3]$. These seeds were spatially separated by at least $12 \mathrm{~mm}(1 \mathrm{FWHM})$. 
Global gray matter volume was calculated by integrating all the modulated voxel values of gray matter segments.

Data analysis

Eight SPM multiple regression models were estimated in order to test, across subjects, the strength of structural covariance of the striatal seeds (four per hemisphere) with the other brain areas. In each regression model, together with the volume of the seed of interest, we included, as confounding covariates, global gray matter, age, gender, and the remaining three striatal seed volumes from the same hemisphere.

To assess for potential interactions of striatal connectivity with gender and age, subjects were divided into four subgroups (male / female - younger / older). The cut-point between younger and older subjects was 30 years, with the aim of providing a relatively balanced distribution of subjects across groups (22 younger males, 18 older males, 28 younger women, and 22 older women) and two age groups that were reasonably representative of our particular range (20-58 years), which, in developmental terms, included early and middle adulthood subjects. We estimated eight additional SPM models similar to those described above, although subjects were divided into these four subgroups. Such models permitted the assessment of first-order (with gender or age) and second-order (with gender and age) interactions of the seed of interest's voxel-wise correlations. Global gray matter and the other three seed volumes from the same hemisphere were introduced as confounding covariates. The analyses were initially restricted to the regions where significant correlations with the seed of interest were observed in the general analyses described above (using small volume correction [SVC] procedures to assess statistical significance), although as interactions of structural covariance with age and gender may also be expected outside these regions, analyses were also performed at the whole-brain level. 
For the assessment of potential asymmetries in structural covariance, four multiple regression models (one for each pair of homologous seeds) were built. In these models, gray matter images were entered twice, in their original orientation and flipped (right hemisphere on the left and vice versa). The models were estimated in such a way that seed volumes from the left hemisphere were correlated against the original images, whereas seed volumes from the right hemisphere were correlated against the flipped images. Therefore, when analyzing covariance differences between left and right seeds, findings located in the left hemisphere corresponded to between-seed differences in ipsilateral connectivity, whilst findings located in the right hemisphere corresponded to between-seed differences in contralateral connectivity. Global gray matter, age, gender, and the other striatal seed volumes were entered as confounding variables.

In all the analyses, variables were sequentially orthogonalized following an iterative GramSchimdt procedure. In each model, the global gray matter volume was always the first to be entered, followed by age and gender (except in the analyses assessing for potential interactions with these variables), the striatal seeds of no interest, and the striatal seed of interest. Following such an approach, we aimed to remove from the seed of interest all the variance shared with the other striatal seeds (as well as with the general confounding factors of global gray matter, age and gender), thus avoiding the inclusion of multiple collinear measurements in the design matrix. The results of such analyses were expected to be maximally specific structural covariance whole-brain patterns. T-statistic maps were generated by assessing the positive correlations of the seed-region of interest with the rest of the brain (voxel-wise). A statistical threshold of $p<0.05$ False Discovery Rate (FDR) corrected was used in all cases. In all the analyses, voxels less than $2 \mathrm{~cm}(\sim 2 \mathrm{FWHM})$ away from the seed of interest were masked out in order to remove correlations that could be induced by smoothing (Mechelli et al. 2005).

Correlations between our seed-regions of interest, including correlations between homologous seeds, were calculated in SPSS v.15 (SPSS Inc., Chicago, IL) controlling for age, gender and global gray matter volume. 


\section{Results}

\section{Caudate seeds}

Our results showed a segregation of the patterns of structural covariance of dorsal and ventral caudate seeds (Fig. 2 and Table 1). Dorsal caudate seeds correlated with the dorso-medial prefrontal regions (medial frontal gyrus). The left dorsal caudate region demonstrated a specific association with the dorsal (rostral) anterior cingulate cortex, whereas the right dorsal caudate region demonstrated a specific association with the right anterior insular cortex. Despite such asymmetries in the pattern of structural covariance, formal testing of correlation asymmetries between the left and right dorsal caudate regions showed no statistically significant differences.

The ventral caudate/accumbens region demonstrated mostly symmetrical structural covariance patterns, with significant correlations detected at the medial orbitofrontal cortex (gyrus rectus), medial thalamus, dorsal (caudal) anterior cingulate cortex, left middle frontal cortex (specifically for the left seed), ventral midbrain (ventral tegmental area (VTA)-substantia nigra (SN) region), and other ventral regions such as the amygdalae, the parahippocampal and fusiform gyri, and the right cerebellar cortex. Asymmetry testing indicated no significant between-hemisphere differences.

\section{Putamen seeds}

Patterns of structural covariance of the dorso-caudal and ventro-rostral putamen were less spatially extended than those of the caudate subregions (see Fig. 2 and Table 1). Both dorsocaudal putamen regions were correlated with the left fusiform gyrus and with discrete thalamic nuclei (left seed with the medial dorsal nucleus, and right seed with the ventral lateral nucleus). The left dorso-caudal putamen region was additionally correlated with the contralateral angular gyrus, hippocampus, and ventro-caudal putamen. This asymmetry in the structural covariance pattern was confirmed by explicitly testing the differences in the structural covariance pattern of the left and right dorso-caudal putamen seeds (see Table 2). 
The ventro-rostral putamen region was correlated with the anterior insula bilaterally, with the right precentral gyrus and the ipsilateral temporal pole. Specifically for the left hemisphere, we observed a significant correlation with the ipsilateral inferior frontal gyrus, and specifically for the right hemisphere, we observed a correlation with the contralateral temporal pole, although direct testing of the asymmetries revealed no significant differences.

Interactions with gender and/or age

When analyses were restricted to the aforementioned significant structural covariance maps, we detected that the correlation between the right ventral caudate and the ipsilateral medial thalamus was stronger in younger than in older individuals (see Fig. S1). At the whole brain level, we observed a significant interaction with age in the structural covariance pattern of the right dorsal caudate region. Specifically, a correlation between this region and the sensorimotor and inferior parietal cortices was observed in older individuals, but not in the younger subgroup (see Fig. 3).

Although no significant interactions with gender were detected, we observed (at the whole-brain level) a second-order interaction between the left dorsal putamen and three clusters located at the right superior temporal gyrus (extending to the right Heschl gyrus), the posterior cingulate cortex, and the caudal portion of left middle temporal gyrus (see Fig. 4). Specifically, within the group of younger subjects, such correlations were significantly stronger in male than in female subjects. This gender effect was not observed in the older subject cohort.

\section{Correlations between seeds}

All homologous seed pairs were significantly correlated. Dorsal caudate seeds were correlated at $r=0.86$, ventral caudate seeds at $r=0.82$, dorsal caudal putamen seeds at $r=0.68$, and the ventral rostral putamen seeds at $r=0.67$ ( $p<0.0005$ in all cases). We also studied the correlations between non-homologous seeds. Collapsed across hemispheres, we observed a 
significant correlation between the ventral caudate and the ventral rostral putamen $(r=0.76$, $p<0.0005)$, and between the dorsal caudal putamen and the ventral rostral putamen $(r=0.48$, $p<0.0005$; see Table S1).

Finally, we conducted an analysis aimed at assessing the relevance of controlling for global gray matter content in structural covariance studies. Within SPSS, we studied the correlation between raw seed values and the average of the voxel values from the regions significantly correlated with each particular seed in the above SPM analyses. From these analyses, we extracted the standardized differences in fit value (sDFFIT) for each subject, which were correlated with global gray matter content. Correlations were significant and positive for all seeds, with fair to moderate $r$ values (from 0.21 to 0.53 ), and $p<0.05$ in all cases, which meant that larger global gray matter content was associated with larger structural covariance values.

\section{Discussion}

We characterized structural covariance patterns of the neostriatum by adopting an anatomical parcellation scheme that has been used in several recent human functional connectivity studies (DiMartino et al. 2008, 2011; Harrison et al. 2009; Kelly et al. 2009; Kwak et al. 2010; Fitzgerald et al. 2011; Sakai et al. 2011). Our results indicate that neostriatal structural covariance networks overlap well with corresponding functional connectivity results, despite being generally more restricted in their cortical distribution, as is also reflected in other previous research (Cohen et al. 2008). We have also shown that such structural covariance patterns are highly symmetrical, with the exception of the dorsal-caudal putamen patterns. Finally, our results suggest that such structural covariance patterns interact with age, thus demonstrating the dynamic nature of the phenomenon of related gray matter content across distant structures.

Dorsal and ventral caudate subregions showed segregated patterns of structural covariance. On the one hand, dorsal caudate was mainly correlated with dorso-medial prefrontal regions, such as the supracallosal anterior cingulate and paracingulate cortices, but also with the right 
anterior insular cortex. On the other hand, the ventral caudate was bilaterally correlated with ventral limbic structures, such as the medial orbitofrontal cortex, the amygdala, and the parahipocampal gyrus. It was also correlated with the ventral aspect of the cingulate gyrus (where most of the limbic inputs to the cingulate are received (Paus 2001), from the amygdala (Barbas and De Olmos 1990) or the ventral striatum (Kunishio and Haber 1994)), and with different subcortical structures such as the medial thalamus, the midbrain or the cerebellum. Importantly, these findings are in general agreement with the traditional cortico-striatal circuit models (Alexander et al., 1986) and with previous studies assessing the functional and structural connectivity of the same striatal territories (Lehéricy et al. 2004; Postuma and Dagher 2006; DiMartino et al. 2008; Draganski et al. 2008).

The dorsal-caudal putamen showed the most restricted structural covariance pattern of all the regions assessed. Besides the expected correlation with the homologous seed, the right dorsalcaudal putamen was only correlated with the left lingual gyrus and the ipsilateral ventral lateral nucleus of the thalamus, while the left dorsal putamen was correlated with the medial dorsal nucleus of the thalamus, and, interestingly, with the right angular gyrus and the right hippocampus, which were the only significant findings in the asymmetry analyses performed between all homologous seed pairs. Although the specific meaning of such lateralization is not clear, it indicates that structural covariance patterns may be modulated by brain asymmetries. Regarding the ventral-rostral putamen, it was bilaterally correlated with the anterior insular cortex and the temporal pole, and with the adjacent neocortical regions of the left inferior frontal and right precentral gyri. Such data are in agreement with previous functional connectivity findings (DiMartino et al. 2008; Harrison et al. 2009), suggesting that the ventral-rostral putamen is related to structures (i.e., anterior insular cortex/frontal operculum) of the ventral-lateral prefrontal system (Price and Drevets 2010). The superior temporal pole is connected to these structures via the uncinate fasciculus (Kier et al. 2004).

Some of the above-mentioned findings deserve further discussion. Within the cortico-striatal circuit models, the thalamus receives information after basal ganglia processing as a final output relay before reaching the cortex, information that is conveyed through specific thalamic 
nuclei as a function of the cortico-striatal loop involved (Middleton and Strick, 2000). In general, our findings are in agreement with this scheme: the ventral caudate was correlated with the medial thalamus (from which projections are sent to the prefrontal cortex), whereas the right dorsal-caudal putamen was correlated with the ventral lateral nucleus of the thalamus, which is connected to premotor and motor cortices and integrates cortical motor information with dorsal striatal output (McFarland and Haber 2000, 2002; Draganski et al. 2008). Nevertheless, our finding of a correlation between the left dorsal-caudal putamen and the dorsal medial thalamus is in apparent conflict with such a scheme. Interestingly, functional connectivity studies have also detected asymmetric correlations between the putamen and the thalamus (Postuma and Dagher 2006), albeit in an opposite manner (i.e., right putamen correlating with medial thalamus and left putamen correlating with more ventro-lateral regions). Such results, most probably caused by the limited spatial resolution of current imaging techniques, may indeed indicate that each striatal territory is connected to different thalamic nuclei, probably due to the substantial amount of collateralization detected at basal ganglia output structures (i.e., the internal and external pallidum and the pars reticulata of the substantia nigra) (Graybiel, 2005).

Correlations between the striatum and the thalamus also differed in their extent. In comparison to the putamen-thalamus correlations, the ventral caudate showed a more extended pattern of structural covariance with the thalamus. In this respect, it is important to point out that the medial thalamus is also a major source of input information to the ventral striatum (Haber and Calzavara 2009). Such reciprocal connections may well be reflected in an increased structural covariance between structures. Furthermore, since our results also showed a correlation between the ventral striatum and the right cerebellar hemisphere, covariance between the ventral striatum and the thalamus may also be modulated by the incoming projections from the dentate nucleus of the cerebellum (conveying output information from the cerebellar cortex), which reach the striatum through a thalamic relay (Bostan and Strick, 2010; Hoshi et al., 2005). In this sense, our results may also suggest that the ventral striatum is a preferential target of such cerebellar projections. 
Also of interest was the correlation observed between the ventral striatum and the region comprising the ventral tegmental area and the substantia nigra, which is in agreement with previous functional connectivity (Harrison et al. 2009) and structural covariance (Cohen et al. 2008) findings. This midbrain region provides extensive dopaminergic input to the ventral striatum and receives even more extensive reciprocal afferences (Haber 2003). Such connections mediate reinforcement-based learning, and provide the substrate for the existence of reciprocal interconnections between the different cortico-striatal loops and the dopaminergic reward system (Graybiel, 2008; Pennartz et al., 2009). The connection between cortico-striatal circuits and the reward system may also rely on the striosomal pathways originating in the dorsal striatum (Crittenden and Graybiel, 2011; Graybiel 2005), although we have not detected any correlations between dorsal striatal regions and the dopamine-containing neurons of the midbrain, most probably because the identification of such pathways remains elusive for current imaging techniques. Nevertheless, considering that striosomes are preferential targets of limbic structures (Crittenden and Graybiel, 2011), it is noteworthy that we have observed a correlation between the anterior insular cortex and dorsal caudate.

Our results are in general agreement with the idea that structural covariance seems to depend on the existence of functional connectivity between regions (Seeley et al. 2009; Zielinski et al. 2010). In this sense, insofar as functional connectivity depends on the existence of direct white matter connections between structures (Damoiseaux and Grecius 2009), structural covariance between functionally connected regions may be mediated by mutually trophic influences of white matter tracts (Mechelli et al. 2005, Wright et al. 1999). Additionally, if no direct anatomical connections exist, structural covariance may also depend on the release of use-related trophic factors, which may link synaptic density and neuropil mass within functionally connected regions (Seeley et al. 2009). Indeed it has been shown that synchronous neural firing promotes synaptogenesis within distributed brain networks (Katz and Shatz 1996; Bi and Poo 1999). However, somewhat unexpectedly, we also observed volumetric correlations between regions that do not appear to have strong functional connectivity. In this case, structural covariance may be expected to rely upon indirect connections between structures. 
Nevertheless, despite the above assumptions, the patterns of structural covariance described here were less extended than those described in functional or structural connectivity assessments of the same (or partially overlapping) striatal territories (Lehéricy et al. 2004; Postuma and Dagher 2006; DiMartino et al. 2008; Draganski et al. 2008; Harrison et al. 2009). Relevantly, we have found no evidence of significant structural covariance between the dorsal caudate and the dorsolateral prefrontal cortex, or between the dorsal-caudal putamen and the motor-premotor cortices. In this respect, it is important to highlight that increased functional connectivity between structures may not only be mediated by structural plasticity, but also by functional plasticity (i.e., Hebbian synaptic plasticity). Functional plasticity is supposed to change synaptic strengths without changing the anatomical connectivity between neurons (Butz et al., 2009), and therefore without promoting structural covariance. Also, complex interactions may exist between functional and structural plasticity. Thus, it has been proposed that, in order to maintain desired levels of activity (i.e., neuronal homeostasis), increases in functional plasticity may be compensated by decreases in the anatomical connectivity between neurons (Butz et al., 2009). This idea may well explain how regions connected at the functional level are not structurally linked.

There are also regional differences in the capacity to show structural plasticity. It has been reported that structural plasticity (i.e., synaptic turnover or rewiring, and changes in the dendritic arborization or spine density) is much more frequently observed in limbic regions than in the prefrontal, motor and sensory cortices (Butz et al., 2009; Kolb et al. 2003; Neufeld et al. 2009). Also, by means of cortical thickness measurements, Lerch et al. (2006) observed that primary motor and sensory cortices show the least structural covariance with other cortical regions. Significantly, such regional differences in the capacity to show structural plasticity seem to be mediated by the presence of particular neurotransmitters, which may act as neurotrophic factors. Structural plasticity in prefrontal and limbic cortices depends on optimal dopamine levels (Neufeld et al. 2009), and, in humans, structural covariance between the neostriatum and the dorsolateral prefrontal cortex is increased in the carriers of a specific haplotype of a protein (DARPP-32) related to molecular mechanisms of dopaminergic neurotransmission (MeyerLindenberg 2009). 
Developmental factors may also play a role in mediating the patterns of structural covariance described in the present study. Similarities in gene expression or similar responses to neurohormonal factors may underpin volumetric correlations across distant regions (Wright et al. 1999). For example, such factors may explain the high correlation values observed for all pairs of homologous seeds. Late neurodevelopmental factors may also play a role therein, as it has been shown that structural covariance develops from infancy to adolescence in cortical functional networks (Zielinski et al. 2010) and in cortico-striatal circuits (Colibazzi et al. 2008). In our adult sample we also observed a significant influence of age, although, in this case, as both increases and decreases of structural covariance with aging. Our results suggest that patterns of structural covariance are dynamic and, although dissimilar aging patterns across structures (Raz et al. 2005) may specifically account for the progressive loss of structural covariance, the interactions with age observed here might be better explained by changes across time in the functional and structural connectivity between structures (Gong et al. 2009; Dosenbach et al. 2010). Specifically, we found an increased structural covariance between the ventral striatum and medial thalamus in younger subjects and an increased structural covariance between the dorsal caudate and somatosensory regions in older subjects. Speculatively, it is tempting to interpret such results within the framework of a ventral-to-dorsal gradient in the cortico-striatal control of behavior. According to this idea, acquired behaviors progressively move from an initial control by ventral cortico-striatal circuits, responsible for goal-oriented behaviors and processing immediate rewards, to a control exerted by dorsal circuits, responsible for habitual modes of action and processing future rewards (Graybiel, 2008; Tanaka et al., 2004).

Furthermore, we also observed an interaction between gender and the structural covariance of the left dorsal-caudal putamen with three cortical regions, although such an interaction also depended on age, which would suggest that, in our sample, age effects were more important modulators of structural covariance than gender-related factors. Nevertheless, gender-related factors (e.g., circulating levels of sex hormones) may affect regional volumes in the adult brain (Witte et al. 2010), and previous studies have shown that structural connectivity within the cortex is indeed modulated by gender (Gong et al. 2009; Yan et al. 2010). Finally, our analyses 
also showed that structural covariance appears to be related to global gray matter content. This may be interpreted as evidence that in larger brains regional gray matter measurements are more related to global gray matter than in smaller brains, resulting in a slightly decreased interregional covariation in the latter. Be that as it may, as we controlled for global gray matter content in all our analyses, our results should not be directly related to this finding.

Our study does, however, have certain limitations. Firstly, although we have tried to interpret our structural covariance findings as related to functional and structural connectivity, it must be said that such an association is, for the time being, speculative. Further research using multimodal imaging assessments (i.e., by means of fMRI, DTI and structural MRI with the same set of subjects) should directly address this issue. Secondly, although our age-effect assessment was based on splitting the sample into two groups to provide a fair representation of the age range of our subjects, age distribution was skewed towards younger subjects, which resulted in a somewhat unbalanced distribution of the subjects across age groups. Future studies specifically assessing changes in structural covariance patterns across different age ranges may be of particular interest in this respect. Bearing such limitations in mind, our findings should be interpreted as further evidence that the gray matter content of distant structures may be correlated and that such correlations are mainly observed within networks of functionally and/or structurally connected regions. Potential factors affecting structural covariance include age, gender and lateralization. In addition to providing a way to explore structural plasticity with neuroimaging techniques, the findings should be of further interest in characterizing structural brain network alterations in basal-ganglia disorders. 


\section{Acknowledgements}

This study was supported in part by the Carlos III Health Institute (PI09/01331 and CP10/00604) and by Agencia de Gestió d'Ajuts Universitaris i de Recerca (AGAUR; 2009SGR1554). Dr. Soriano-Mas is funded by a Miguel Servet contract from the Carlos III Health Institute (CP10/00604). Dr. Harrison is supported by a National Health and Medical Research Council of Australia (NHMRC) Clinical Career Development Award (I.D. 628509). We thank Mr. Gerald Fannon for revising the manuscript.

\section{Financial Disclosures}

The authors declare that they have no conflict of interest. 


\section{References}

Alexander GE, DeLong MR, Strick PL (1986) Parallel organization of functionally segregated circuits linking basal ganglia and cortex. Annu Rev Neurosci 9:357-381

Allen JS, Damasio H, Grabowski TJ (2002) Normal neuroanatomical variation in the human brain: an MRI-volumetric study. Am J Phys Anthropol 118:341-358

Andrews TJ, Halpern SD, Purves D (1997) Correlated size variations in human visual cortex, lateral geniculate nucleus, and optic tract. J Neurosci 17:2859-2868

Ashburner J, Friston KJ (2005) Unified segmentation. Neuroimage 26:839-851

Barbas H, De Olmos J (1990) Projections from the amygdala to basoventral and mediodorsal prefrontal regions in the rhesus monkey. J Comp Neurol 300:549-571

Bi G, Poo M (1999) Distributed synaptic modification in neural networks induced by patterned stimulation. Nature 401:792-796

Brett MA, Anton JL, Valabregue R, Poline J (2002) Region of interest analysis using an SPM toolbox. Neuroimage 16:497 (abstract)

Bostan AC, Strick PL (2010) The cerebellum and basal ganglia are interconnected. Neuropsychol Rev 20:261-270

Butz M, Wörgötter F, van Ooyen A (2009) Activity-dependent structural plasticity. Brain Res Rev 60:287-305

Cardoner N, Soriano-Mas C, Pujol J, Alonso P, Harrison BJ, Deus J, Hernández-Ribas R, Menchón JM, Vallejo J (2007) Brain structural correlates of depressive comorbidity in obsessive-compulsive disorder. Neuroimage 38:413-421

Caviness VS Jr, Lange NT, Makris N, Herbert MR, Kennedy DN (1999) MRI-based brain volumetrics: emergence of a developmental brain science. Brain Dev 21:289-295

Cohen MX, Lombardo MV, Blumenfeld RS (2008) Covariance-based subdivision of the human striatum using T1-weighted MRI. Eur J Neurosci 27:1534-1546

Colibazzi T, Zhu H, Bansal R, Schultz RT, Wang Z, Peterson BS (2008) Latent volumetric structure of the human brain: Exploratory factor analysis and structural equation modeling of gray matter volumes in healthy children and adults. Hum Brain Mapp 29:1302-1312 
Crittenden JR, Graybiel AM (2011) Basal Ganglia disorders associated with imbalances in the striatal striosome and matrix compartments. Front Neuroanat 5:59

Damoiseaux JS, Greicius MD (2009) Greater than the sum of its parts: a review of studies combining structural connectivity and resting-state functional connectivity. Brain Struct Funct 213:525-533

Di Martino A, Kelly C, Grzadzinski R, Zuo XN, Mennes M, Mairena MA, Lord C, Castellanos FX, Milham MP (2011) Aberrant striatal functional connectivity in children with autism. Biol Psychiatry 69:847-856

Di Martino A, Scheres A, Margulies DS, Kelly AM, Uddin LQ, Shehzad Z, Biswal B, Walters JR, Castellanos FX, Milham MP (2008) Functional connectivity of human striatum: a resting state FMRI study. Cereb Cortex 18:2735-2747

Dosenbach NU, Nardos B, Cohen AL, Fair DA, Power JD, Church JA, Nelson SM, Wig GS, Vogel AC, Lessov-Schlaggar CN, Barnes KA, Dubis JW, Feczko E, Coalson RS, Pruett JR Jr, Barch DM, Petersen SE, Schlaggar BL (2010) Prediction of individual brain maturity using fMRI. Science 329:1358-1361

Draganski B, Gaser C, Busch V, Schuierer G, Bogdahn U, May A (2004) Neuroplasticity: changes in grey matter induced by training. Nature 427:311-312

Draganski B, Kherif F, Klöppel S, Cook PA, Alexander DC, Parker GJ, Deichmann R, Ashburner J, Frackowiak RS (2008) Evidence for segregated and integrative connectivity patterns in the human Basal Ganglia. J Neurosci 28:7143-7152

Fitzgerald KD, Welsh RC, Stern ER, Angstadt M, Hanna GL, Abelson JL, Taylor SF (2011) Developmental alterations of frontal-striatal-thalamic connectivity in obsessivecompulsive disorder. J Am Acad Child Adolesc Psychiatry 50:938-948 e3

Giedd JN, Snell JW, Lange N, Rajapakse JC, Casey BJ, Kozuch PL, Vaituzis AC, Vauss YC, Hamburger SD, Kaysen D, Rapoport JL (1996) Quantitative magnetic resonance imaging of human brain development: ages 4-18. Cereb Cortex 6:551-560

Gong G, Rosa-Neto P, Carbonell F, Chen ZJ, He Y, Evans AC (2009) Age- and gender-related differences in the cortical anatomical network. J Neurosci 29:15684-15693 
Good CD, Johnsrude IS, Ashburner J, Henson RN, Friston KJ, Frackowiak RS (2001) A voxelbased morphometric study of ageing in 465 normal adult human brains. Neuroimage 14(1 Pt 1):21-36

Graybiel AM (2005) The basal ganglia: learning new tricks and loving it. Curr Opin Neurobiol $15: 638-644$

Graybiel AM (2008) Habits, rituals, and the evaluative brain. Annu Rev Neurosci 31:359-387

Haber SN (2003) The primate basal ganglia: parallel and integrative networks. J Chem Neuroanat 26:317-330

Haber SN, Calzavara R (2009) The cortico-basal ganglia integrative network: the role of the thalamus. Brain Res Bull 78:69-74

Harrison BJ, Soriano-Mas C, Pujol J, Ortiz H, López-Solà M, Hernández-Ribas R, Deus J, Alonso P, Yücel M, Pantelis C, Menchon JM, Cardoner N (2009) Altered corticostriatal functional connectivity in obsessive-compulsive disorder. Arch Gen Psychiatry 66:11891200

Hoshi E, Tremblay L, Féger J, Carras PL, Strick PL (2005) The cerebellum communicates with the basal ganglia. Nat Neurosci 8:1491-1493

Huttenlocher PR (1979) Synaptic density in human frontal cortex - developmental changes and effects of aging. Brain Res 163:195-205

Kaspárek T, Marecek R, Schwarz D, Prikryl R, Vanícek J, Mikl M, Cesková E (2010) Sourcebased morphometry of gray matter volume in men with first-episode schizophrenia. Hum Brain Mapp 31:300-310

Katz LC, Shatz CJ (1996) Synaptic activity and the construction of cortical circuits. Science 274:1133-1138

Kelly C, de Zubicaray G, Di Martino A, Copland DA, Reiss PT, Klein DF, Castellanos FX, Milham MP, McMahon K (2009) L-dopa modulates functional connectivity in striatal cognitive and motor networks: a double-blind placebo-controlled study. J Neurosci 29:7364-7378

Kennedy DN, Lange N, Makris N, Bates J, Meyer J, Caviness VS Jr (1998) Gyri of the human neocortex: an MRI-based analysis of volume and variance. Cereb Cortex 8:372-384 
Kier EL, Staib LH, Davis LM, Bronen RA (2004) MR imaging of the temporal stem: anatomic dissection tractography of the uncinate fasciculus, inferior occipitofrontal fasciculus, and Meyer's loop of the optic radiation. AJNR Am J Neuroradiol 25:677-691

Kolb B, Gorny G, Söderpalm AH, Robinson TE (2003) Environmental complexity has different effects on the structure of neurons in the prefrontal cortex versus the parietal cortex or nucleus accumbens. Synapse 48:149-153

Kunishio K, Haber SN (1994) Primate cingulostriatal projection: limbic striatal versus sensorimotor striatal input. J Comp Neurol 350:337-356

Kwak Y, Peltier S, Bohnen NI, Müller ML, Dayalu P, Seidler RD (2010) Altered resting state cortico-striatal connectivity in mild to moderate stage Parkinson's disease. Front Syst Neurosci 4:143

Lawrence AD, Sahakian BJ, Robbins TW (1998) Cognitive functions and corticostriatal circuits: insights from Huntington's disease. Trends Cogn Sci 2:379-388

Lehéricy S, Ducros M, Van de Moortele PF, Francois C, Thivard L, Poupon C, Swindale N, Ugurbil K, Kim DS (2004) Diffusion tensor fiber tracking shows distinct corticostriatal circuits in humans. Ann Neurol 55:522-529

Lerch JP, Worsley K, Shaw WP, Greenstein DK, Lenroot RK, Giedd J, Evans AC (2006) Mapping anatomical correlations across cerebral cortex (MACACC) using cortical thickness from MRI. Neuroimage 31:993-1003

May A, Gaser C (2006) Magnetic resonance-based morphometry: a window into structural plasticity of the brain. Curr Opin Neurol 19:407-411

McFarland NR, Haber SN (2000) Convergent inputs from thalamic motor nuclei and frontal cortical areas to the dorsal striatum in the primate. J Neurosci 20:3798-3813

McFarland NR, Haber SN (2002) Thalamic relay nuclei of the basal ganglia form both reciprocal and nonreciprocal cortical connections, linking multiple frontal cortical areas. J Neurosci $22: 8117-8132$

Mechelli A, Friston KJ, Frackowiak RS, Price CJ (2005) Structural covariance in the human cortex. J Neurosci 25:8303-8310

Meyer-Lindenberg A (2009) Neural connectivity as an intermediate phenotype: brain networks under genetic control. Hum Brain Mapp 30:1938-1946 
Middleton FA, Strick PL (2000) Basal ganglia output and cognition: evidence from anatomical, behavioral, and clinical studies. Brain Cogn 42:183-200

Mitelman SA, Brickman AM, Shihabuddin L, Newmark R, Chu KW, Buchsbaum MS (2005a) Correlations between MRI-assessed volumes of the thalamus and cortical Brodmann's areas in schizophrenia. Schizophr Res 75:265-281

Mitelman SA, Buchsbaum MS, Brickman AM, Shihabuddin L (2005b) Cortical intercorrelations of frontal area volumes in schizophrenia. Neuroimage 27:753-770

Mitelman SA, Shihabuddin L, Brickman AM, Buchsbaum MS (2005c) Cortical intercorrelations of temporal area volumes in schizophrenia. Schizophr Res 76:207-229

Modinos G, Vercammen A, Mechelli A, Knegtering H, McGuire PK, Aleman A (2009) Structural covariance in the hallucinating brain: a voxel-based morphometry study. J Psychiatry Neurosci 34:465-469

Nakano K, Kayahara T, Tsutsumi T, Ushiro H (2000) Neural circuits and functional organization of the striatum. J Neurol 247 (Suppl 5):V1-V15

Neufeld J, Teuchert-Noodt G, Grafen K, Winter Y, Witte AV (2009) Synapse plasticity in motor, sensory, and limbo-prefrontal cortex areas as measured by degrading axon terminals in an environment model of gerbils (Meriones unguiculatus). Neural Plast 2009:281561

Paus T (2001) Primate anterior cingulate cortex: where motor control, drive and cognition interface. Nat Rev Neurosci 2:417-424

Pennartz CM, Berke JD, Graybiel AM, Ito R, Lansink CS, van der Meer M, Redish AD, Smith KS, Voorn P (2009) Corticostriatal Interactions during Learning, Memory Processing, and Decision Making. J Neurosci 29:12831-12838

Portas CM, Goldstein JM, Shenton ME, Hokama HH, Wible CG, Fischer I, Kikinis R, Donnino R, Jolesz FA, McCarley RW (1998) Volumetric evaluation of the thalamus in schizophrenic male patients using magnetic resonance imaging. Biol Psychiatry 43:649-659

Postuma RB, Dagher A (2006) Basal ganglia functional connectivity based on a meta-analysis of 126 positron emission tomography and functional magnetic resonance imaging publications. Cereb Cortex 16:1508-1521

Price JL, Drevets WC (2010) Neurocircuitry of mood disorders. Neuropsychopharmacology $35: 192-216$ 
Pujol J, Soriano-Mas C, Alonso P, Cardoner N, Menchón JM, Deus J, Vallejo J (2004) Mapping structural brain alterations in obsessive-compulsive disorder. Arch Gen Psychiatry $61: 720-730$

Pujol J, Vendrell P, Junqué C, Martí-Vilalta JL, Capdevila A (1993) When does human brain development end? Evidence of corpus callosum growth up to adulthood. Ann Neurol $34: 71-75$

Raz N, Lindenberger U, Rodrigue KM, Kennedy KM, Head D, Williamson A, Dahle C, Gerstorf D, Acker JD (2005) Regional brain changes in aging healthy adults: general trends, individual differences and modifiers. Cereb Cortex 15:1676-1689

Sakai Y, Narumoto J, Nishida S, Nakamae T, Yamada K, Nishimura T, Fukui K (2011) Corticostriatal functional connectivity in non-medicated patients with obsessivecompulsive disorder. Eur Psychiatry 26:463-469

Seeley WW, Crawford RK, Zhou J, Miller BL, Greicius MD (2009) Neurodegenerative diseases target large-scale human brain networks. Neuron 62:42-52

Tanaka SC, Doya K, Okada G, Ueda K, Okamoto Y, Yamawaki S (2004) Prediction of immediate and future rewards differentially recruits cortico-basal ganglia loops. Nat Neurosci 7:887-893

Witte AV, Savli M, Holik A, Kasper S, Lanzenberger R (2010) Regional sex differences in grey matter volume are associated with sex hormones in the young adult human brain. Neuroimage 49:1205-1212

Wright IC, Sharma T, Ellison ZR, McGuire PK, Friston KJ, Brammer MJ, Murray RM, Bullmore ET (1999) Supra-regional brain systems and the neuropathology of schizophrenia. Cereb Cortex 9:366-378

Xu L, Groth KM, Pearlson G, Schretlen DJ, Calhoun VD (2009) Source-based morphometry: the use of independent component analysis to identify gray matter differences with application to schizophrenia. Hum Brain Mapp 30:711-724

Yakovlev PI, Lecours AR (1967) The myelogenetic cycles of regional maturation of the brain. In: Minkowski A (ed) Regional development of the brain in early life. Blackwell Scientific, Oxford, pp 3-70 
Yan C, Gong G, Wang J, Wang D, Liu D, Zhu C, Chen ZJ, Evans A, Zang Y, He Y (2010) Sexand brain size-related small-world structural cortical networks in young adults: a DTI tractography study. Cereb Cortex 21:449-458

Zielinski BA, Gennatas ED, Zhou J, Seeley WW (2010) Network-level structural covariance in the developing brain. Proc Natl Acad Sci U S A 107:18191-18196 
Table 1 Regions showing significant structural covariance with each of the neostriatal seeds

\begin{tabular}{|c|c|c|c|c|c|c|}
\hline$x$ & $\mathrm{y}$ & $z^{a}$ & $\mathrm{t}$ value & $\begin{array}{c}p \text { value } \\
\text { (FDR corrected) }\end{array}$ & $\begin{array}{l}\text { Cluster extent } \\
\text { (voxels) }\end{array}$ & Anatomical location \\
\hline \multicolumn{7}{|c|}{ Left Dorsal Caudate } \\
\hline 14 & 15 & 10 & 15.52 & $<0.0005$ & 3501 & Right Caudate \\
\hline 2 & 36 & 12 & 3.77 & 0.030 & 3220 & Right Anterior Cingulate Cortex \\
\hline-4 & 12 & 42 & 3.55 & 0.044 & & Left Anterior Cingulate Cortex \\
\hline-1 & 42 & 27 & 3.51 & 0.05 & & Left Medial Frontal Gyrus \\
\hline \multicolumn{7}{|c|}{ Right Dorsal Caudate } \\
\hline-11 & 14 & 8 & 17.29 & $<0.0005$ & 3069 & Left Caudate \\
\hline 37 & 15 & 6 & 4.38 & 0.004 & 1564 & Right Insula \\
\hline 40 & 11 & -17 & 3.67 & 0.037 & 421 & Right Insula \\
\hline-1 & 32 & 39 & 3.59 & 0.046 & 1273 & Left Medial Frontal Gyrus \\
\hline \multicolumn{7}{|c|}{ Left Ventral Caudate } \\
\hline 11 & 10 & -9 & 10.17 & $<0.0005$ & 13578 & Right Ventral Striatum \\
\hline 13 & 11 & 6 & 7.85 & $<0.0005$ & & Right Caudate \\
\hline-5 & 1 & 7 & 5.58 & $<0.0005$ & & Left Medial Thalamus \\
\hline-5 & -9 & -10 & 5.51 & $<0.0005$ & & Left Midbrain (VTA/SN region) \\
\hline-3 & 27 & -15 & 4.75 & $<0.0005$ & & Left Gyrus Rectus \\
\hline-19 & -3 & -15 & 4.70 & $<0.0005$ & & Left Amygdala \\
\hline 17 & -7 & -11 & 3.89 & 0.006 & & Right Amygdala \\
\hline-12 & 3 & 9 & 9.50 & $<0.0005$ & 709 & Left Caudate \\
\hline-22 & 8 & 50 & 4.34 & 0.001 & 334 & Left Middle Frontal Gyrus \\
\hline-32 & -39 & -21 & 4.01 & 0.004 & 460 & Left Fusiform Gyrus \\
\hline 44 & -71 & -30 & 3.87 & 0.006 & 1530 & Right Cerebellar Hemisphere \\
\hline-2 & 10 & 32 & 3.83 & 0.007 & 1619 & Left Mid Cingulate Cortex \\
\hline \multicolumn{7}{|c|}{ Right Ventral Caudate } \\
\hline-9 & 9 & -6 & 12.75 & $<0.0005$ & 18209 & Left Ventral Striatum \\
\hline-9 & 6 & 6 & 6.36 & $<0.0005$ & & Left Caudate \\
\hline-1 & 25 & -14 & 6.00 & $<0.0005$ & & Left Gyrus Rectus \\
\hline-16 & 0 & -13 & 5.36 & $<0.0005$ & & Left Amygdala \\
\hline 3 & -2 & 5 & 5.30 & $<0.0005$ & & Right Medial Thalamus \\
\hline-5 & -7 & -10 & 5.15 & $<0.0005$ & & Left Midbrain (Ventral Tegmantal Area/Substantia Nigra) \\
\hline 14 & 9 & 11 & 6.50 & $<0.0005$ & 391 & Right Caudate \\
\hline 23 & -43 & -13 & 4.40 & 0.003 & 2090 & Right Parahipocampal Gyrus \\
\hline 1 & 2 & 35 & 3.72 & 0.017 & 1180 & Right Mid Cingulate Cortex \\
\hline 49 & -65 & -28 & 3.50 & 0.030 & 1163 & Right Cerebellar Hemisphere \\
\hline-3 & 26 & 24 & 3.44 & 0.035 & 273 & Left Anterior Cingulate Cortex \\
\hline-21 & -36 & -15 & 3.36 & 0.043 & 499 & Left Parahipocampal Gyrus \\
\hline \multicolumn{7}{|c|}{ Left Dorsal-Caudal Putamen } \\
\hline 29 & 0 & 0 & 8.65 & $<0.0005$ & 5285 & Right Putamen \\
\hline 34 & -8 & -14 & 3.73 & 0.021 & & Right Hippocampus \\
\hline 37 & -58 & 33 & 3.79 & 0.017 & 64 & Right Angular Gyrus \\
\hline-25 & -61 & -11 & 3.77 & 0.018 & 157 & Left Lingual Gyrus \\
\hline-7 & -17 & 1 & 3.65 & 0.03 & 141 & Left Thalamus (Medial dorsal nucleus) \\
\hline \multicolumn{7}{|c|}{ Right Dorsal-Caudal Putamen } \\
\hline-26 & -5 & 3 & 8.82 & $<0.0005$ & 2932 & Left Putamen \\
\hline-26 & -64 & -9 & 3.99 & 0.012 & 270 & Left Lingual Gyrus \\
\hline 9 & -12 & 4 & 3.67 & 0.032 & 30 & Right Thalamus (Ventral lateral nucleus) \\
\hline \multicolumn{7}{|c|}{ Left Ventral-Rostral Putamen } \\
\hline 22 & 15 & -1 & 10.11 & $<0.0005$ & 5498 & Right Putamen \\
\hline 35 & 16 & -4 & 4.08 & 0.005 & & Right Anterior Insula \\
\hline-41 & 11 & 9 & 5.22 & $<0.0005$ & 3758 & Left Anterior Insula \\
\hline-48 & 28 & -8 & 4.28 & 0.003 & & Left Inferior Frontal Gyrus (Pars Orbitalis) \\
\hline 49 & 0 & 20 & 4.42 & 0.002 & 294 & Right Precentral Gyrus \\
\hline-49 & 7 & -23 & 3.48 & 0.028 & 262 & Left Superior Temporal Pole \\
\hline \multicolumn{7}{|c|}{ Right Ventral-Rostral Putamen } \\
\hline-20 & 13 & -3 & 10.19 & $<0.0005$ & 17071 & Left Putamen \\
\hline-47 & 8 & -24 & 4.30 & 0.003 & & Left Superior Temporal Pole \\
\hline-42 & 7 & 8 & 4.11 & 0.005 & & Left Anterior Insula \\
\hline 38 & 4 & -25 & 4.41 & 0.002 & 1343 & Right Superior Temporal Pole \\
\hline 49 & 2 & 20 & 3.99 & 0.007 & 246 & Right Precentral Gyrus \\
\hline 37 & 5 & -5 & 3.74 & 0.012 & 239 & Right Anterior Insula \\
\hline
\end{tabular}

${ }^{a} \mathrm{x}, \mathrm{y}, \mathrm{z}$ coordinates are reported in standard Montreal Neurological Institute (MNI) space 
Table 2 Significant asymmetries in the patterns of structural covariance

\begin{tabular}{|c|c|c|c|c|c|c|}
\hline $\mathrm{X}$ & $y$ & $z^{a}$ & $\mathrm{t}$ value & $p$ value $^{b}$ & Direction of findings & Anatomical location \\
\hline \multicolumn{7}{|c|}{ Dorso-Caudal Putamen (Contralateral Correlations) } \\
\hline 33 & -5 & -7 & 4.25 & $<0.0005$ & Left seed > Right seed & Right Ventral-Caudal Putamen \\
\hline 26 & -8 & -19 & 3.40 & $<0.0005$ & Left seed $>$ Right seed & Right Hippocampus \\
\hline 32 & -61 & 34 & 3.07 & 0.001 & Left seed > Right seed & Right Angular Gyrus \\
\hline
\end{tabular}

${ }^{a} \mathrm{x}, \mathrm{y}, \mathrm{z}$ coordinates are reported in standard Montreal Neurological Institute (MNI) space

${ }^{\mathrm{b}}$ FDR corrected 
Figure Legends.

Fig. 1 Seed placements overlaid on high resolution coronal sections. ' $y$ ' denotes the anteriorposterior coordinate in standard Montreal Neurological Institute (MNI) space. Right hemisphere is displayed on the right. DC, Dorsal Caudate; DCP, Dorsal-caudal Putamen; VC, Ventral Caudate; VRP, Ventral-rostral Putamen

Fig. 2 Regions of significant correlation with left and right hemisphere seeds overlaid on a high resolution anatomical image. Voxels with $p_{\mathrm{FDR}}<0.05$ are displayed. In axial and coronal sections, right hemisphere is displayed on the right. DC, Dorsal Caudate seed; DCP, Dorsal-Caudal Putamen seed; VC, Ventral Caudate Seed; VRP, Ventral-Rostral Putamen seed. See text and Table 1 for details

Fig. 3 Regions where structural covariance with right Dorsal Caudate seed interacted with age group. a. Peak coordinates were located at the right postcentral gyrus (extending to the inferior parietal lobe and to the precentral gyrus; $x, y, z=50,-26,52 ; t=5.12$ ), the left postcentral gyrus $(x, y, z=-42,-23,44 ; t=4.62)$, and the left inferior parietal lobe $(x, y, z=-41,-51,46 ; t=4.52)$. Voxels with $p_{\mathrm{FDR}}<0.05$ are displayed. Color bar represents $t$ value. $\mathrm{R}$ indicates the right hemisphere. $\mathbf{b}$. Plot displaying the correlations (in younger and older subjects) between the adjusted volumes of the right Dorsal Caudate seed and the right somatosensory cortex (younger subjects: $r=-0.165$; $p>0.05$; older subjects: $r=0.507 ; p=0.001$ )

Fig. 4 Regions where structural covariance with left Dorsal-Caudal Putamen seed interacted with gender, specifically in younger subjects. a. Peak coordinates were located at the right superior temporal gyrus $(x, y, z=62,-3,-7 ; t=5.04)$, the posterior cingulate cortex $(x, y, z=-9,-37$, $34 ; t=4.24)$, and the left caudal middle temporal gyrus $(x, y, z=-50,-47,-3 ; t=4.74)$. Voxels with $p_{\mathrm{FDR}}<0.05$ are displayed. Color bar represents $\mathrm{t}$ value. $\mathrm{R}$ indicates the right hemisphere. $\mathbf{b}$. Plot displaying the correlations (in younger and older subjects) between the adjusted volumes of the left Dorsal-Caudal Putamen seed and the right superior temporal gyrus, in men (left) and 
women (right) (younger subjects (men): $r=0.506 ; p=0.016$; younger subjects (women): $r=-0.199$; $p>0.05$; older subjects (men): $r=-0.425 ; p=0.024$; older subjects (women): $r=0.184 ; p>0.05$ )
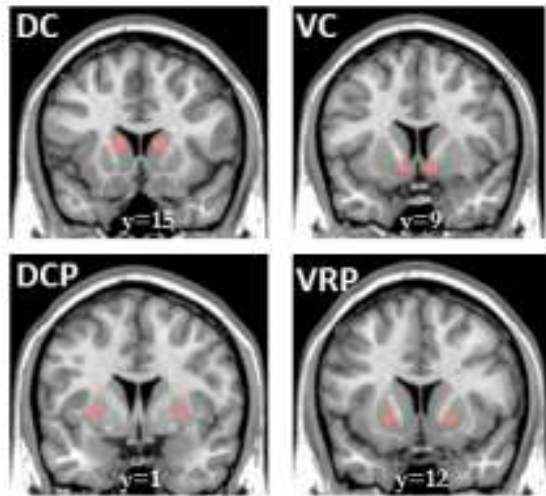


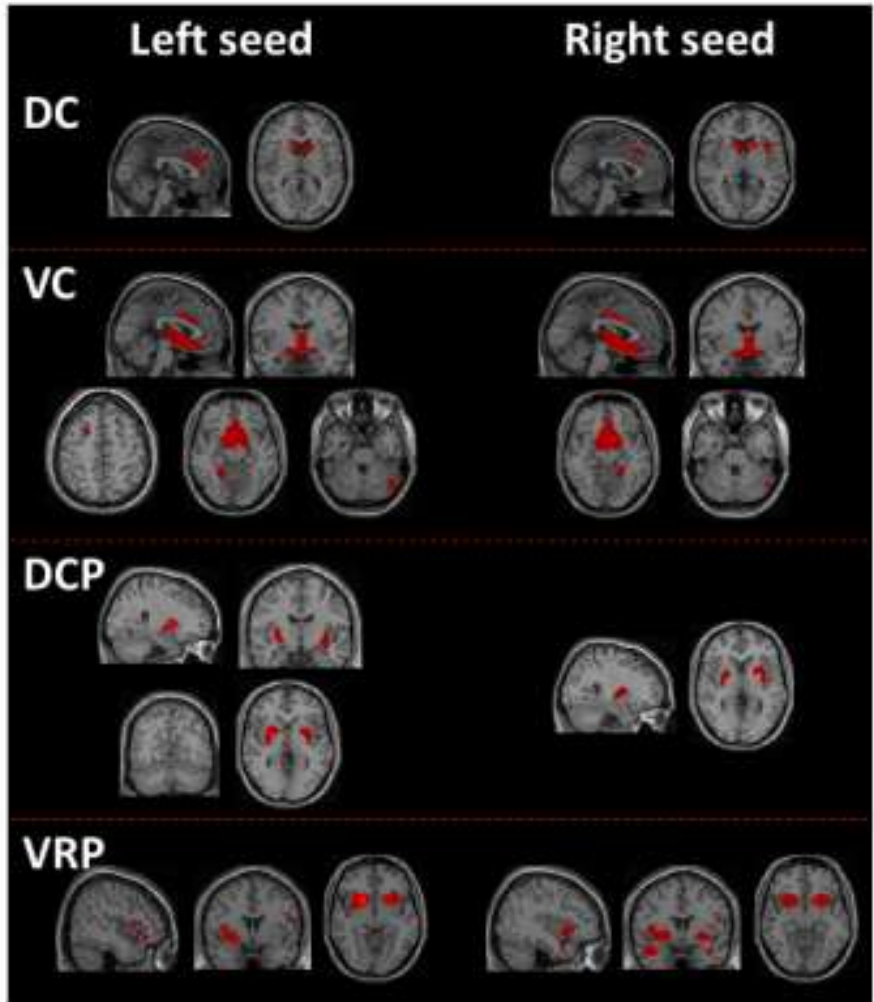

a.

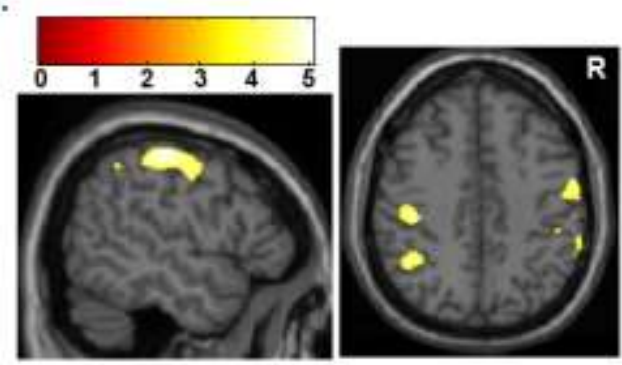

b.

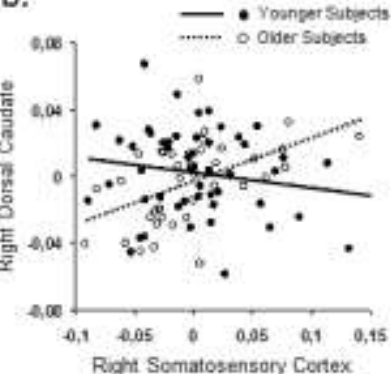




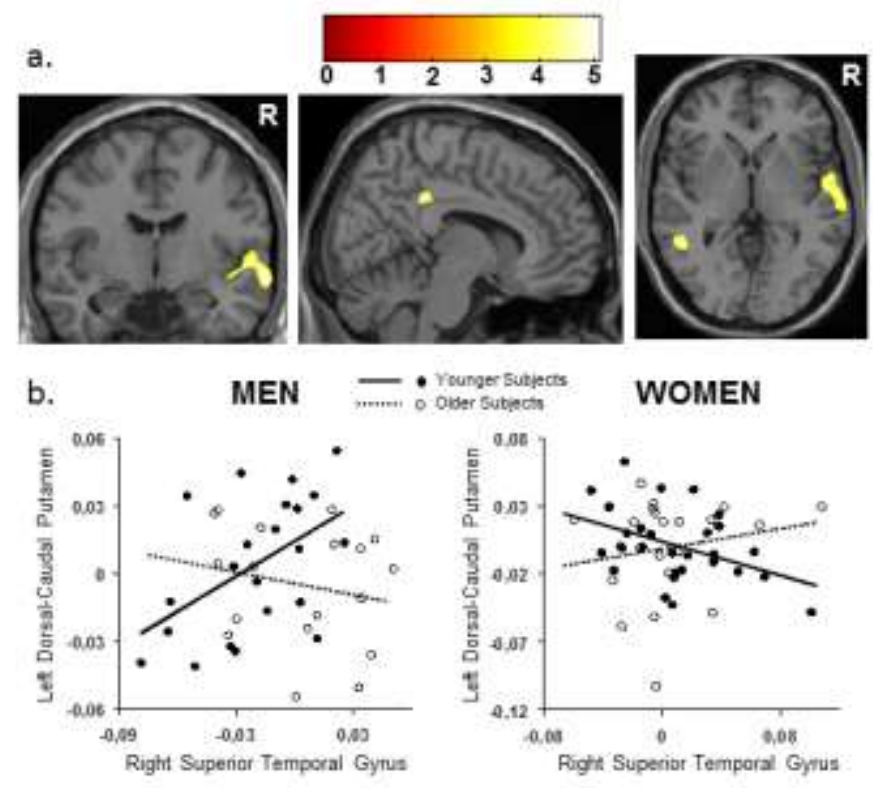

